\title{
Prevention Therapy for Breast Cancer: How Can We Do Better?
}

\author{
Marie E. Wood, MD ${ }^{1}$, Melissa Cuke, MSc ${ }^{1}$, and Isabelle Bedrosian, MD $^{2}$ \\ ${ }^{1}$ Hematology Oncology Division, University of Vermont Larner College of Medicine, Burlington, VT; ${ }^{2}$ University of \\ Texas, MD Anderson Cancer Center, Houston, TX
}

Despite significant advances in therapy for breast cancer, it remains the most frequently diagnosed cancer and leading cause of death among women worldwide, ${ }^{1}$ thus underscoring the need for prevention. For many women, the presence of known risk factors, coupled with the availability of proven risk-reducing agents, provides significant opportunity for prevention. The study in this issue by Flanagan and colleagues investigates the uptake of prevention therapy for women with different risk factors, including benign breast disease, family history of breast and/or ovarian cancer, mutation in a known breast cancerassociated gene, or a history of radiation therapy to the chest prior to age 30 years. ${ }^{2}$ This was a retrospective review of a prospectively accrued cohort of 1506 high-risk women at Memorial Sloan Kettering Cancer Center. The cohort was weighted toward benign breast disease, with $96 \%$ of subjects having a biopsy showing either atypia, atypical ductal hyperplasia (ADH), atypical lobular hyperplasia (ALH), or lobular carcinoma in situ (LCIS). Investigators examined several sources for clarification of prevention therapy use and/or reasons for no use and found that $24 \%$ of women had used or were using prevention therapy. They were able to identify reasons for lack of use in only $50 \%$ of non-users, finding fear of adverse effects to be the most common reason for refusal. This study has several clinically significant findings. First, low uptake of prevention therapy, and, surprisingly, low uptake for women with some form of benign breast disease. Second, lack of documentation of a discussion of prevention therapy for half of the women not taking prevention therapy.

(C) Society of Surgical Oncology 2019

First Received: 28 January 2019;

Published Online: 23 February 2019

M. E. Wood, MD

e-mail: marie.wood@uvm.edu
Lastly, and maybe most importantly, the majority of women who started prevention therapy completed 5 years, despite fear of adverse effects being the most common patient-related barrier to use.

The core finding of low uptake of prevention therapy among high-risk women is not new, however the lack of uptake among women with some form of atypia or LCIS deserves comment. As the authors point out, several agents have been shown to prevent breast cancer. ${ }^{3-5}$ The effects of these agents are actually greater than those used to lower cholesterol for the prevention of cardiovascular disease. ${ }^{6}$ The group that may benefit most from these agents are women with atypical hyperplasia and/or LCIS. The National Surgical Adjuvant Breast and Bowel Project (NSABP) P1 trial demonstrated that women with atypical hyperplasia had an $86 \%$ reduction in risk for breast cancer with tamoxifen, while women with LCIS received a $56 \%$ reduction in risk; ${ }^{7}$ this effect was long-lasting. ${ }^{8}$ The aromatase inhibitors have also shown significant effects for women with atypical hyperplasia or LCIS. ${ }^{5,9}$ Given these efficacy data, and given the prevalence of women diagnosed with atypia and lobular neoplasia, efforts to increase the utilization of chemoprevention in this particular subset of women is of critical importance to reduce the incidence of breast cancer.

The authors provide interesting new insights that could inform novel strategies to enhance the use of prevention therapy. First, half of the women in the current study who were not taking prevention therapy (38\% of the overall cohort) did not have a documented discussion of prevention therapy. Provider recommendation has been shown to be important for the uptake of many prevention strategies, including breast cancer prevention therapy, ${ }^{10,11}$ and the quality of that recommendation is important. ${ }^{12,13}$ Providers are busy and primary care providers in particular have increasingly less time to address all important issues, thus discussions and recommendations around prevention 
therapy may be overlooked. Implementation of a systemlevel approach (where providers are given feedback on their rates of recommendation and prescription of risk-reducing drugs to high-risk women) has been reported to significantly increase both provider recommendation rates and uptake of prevention therapy. ${ }^{14}$ With the rapid adoption of electronic health records (EHRs) across medical practices, the implementation of similar systems-based strategies through the electronic health portal may provide one opportunity to improve on current rates of utilization of prevention therapy. A second important observation by Flanagan and colleagues is that almost two-thirds of women who had used a prevention agent had completed the full 5 years. These data suggest that all efforts should be made to encourage at-risk women to initiate therapy. A key component of these efforts is how the message of prevention therapy is delivered. While often used by providers, the term 'chemoprevention' has been shown to be a barrier to uptake of prevention therapy, ${ }^{15}$ and both national and international groups now recommend that this term be discontinued. ${ }^{16,17}$ While several alternate phrases have been proposed, in our opinion the phrase 'prevention therapy' may be the most acceptable, while accurately conveying the intent of treatment.

Beyond these efforts to improve communication with patients and improve patient compliance with current guidelines for preventative therapy, research efforts are needed to continue to refine the population most likely to benefit. Many of our current algorithms to identify at-risk populations perform well at the population level but less so at an individual level. Additionally, while often higher than the population average, the magnitude of risk determined by these models is low in absolute value, making it harder for women to see the benefit of taking medications with fairly substantial adverse effects. Therefore, there is a great need for a more personalized and precise risk assessment. New opportunities in this regard include genome-wide association studies that have identified $>100$ single nucleotide polymorphisms (SNPs) associated with breast cancer risk. These SNPs have now been incorporated into polygenic risk scores (PRS) and several groups have shown that these PRS perform better than traditional models for risk prediction. The ability of a PRS to improve risk assessment is promising, however validation in both genetically and ethnically diverse populations is needed. Other novel classes of biomarkers, such as microRNAs, may capture clinically relevant information based on epigenetic regulation of gene expression. While progress is being made towards more precise risk assessment, rigorous validation in relevant populations is necessary before widespread clinical use.
A second challenge for preventative therapy is that current prevention agents (selective estrogen receptor modulators and aromatase inhibitors) only reduce the risk of estrogen receptor-positive $(\mathrm{ER}+)$ breast cancer, ${ }^{2}$ and only tamoxifen is available for premenopausal women, providing limited choices for premenopausal women and no current options for ER-negative (ER-) cancers. Prevention therapies with lower toxicities that reduce the rates of both ER + and ER - disease are needed. There are emerging data regarding the prevention properties of several agents (statins, aspirin, vitamin D, metformin, bisphosphonates). ${ }^{17}$ These agents are interesting as they generally have lower toxicities, show promise in the reduction of both ER+ and ER- disease, and are often approved for use in other diseases (both cancer and noncancer). Randomized clinical trials will need to be completed to confirm the findings of biological and epidemiological studies that suggest prevention effects. Given the widespread use of some of these agents (i.e. statins, aspirin, vitamin D), significant consideration to trial design is needed. Focusing on high-risk populations (such as those identified in the current study) and use of biomarker endpoints may avoid costly and long follow-up associated with randomized clinical trials using cancer endpoints.

In summary, the study by Flanagan et al. ${ }^{2}$ highlights missed opportunities for preventing breast cancer. We may be able to do better by harnessing the EHRs, more precise assessment of risk, and identification of low-toxicity agents that are effective for the prevention of ER- breast cancer and useful for premenopausal women.

\section{REFERENCES}

1. Siegel RL, Miller KD, Jemal A. Cancer statistics, 2018. $C A$ Cancer J Clin. 2018;68(1):7-30.

2. Flanagan MR, Zabor EC, Stempel M, Mangino DA, Morrow M, Pilewskie ML. Chemoprevention uptake for breast cancer risk reduction varies by risk factor. Ann Surg Oncol. 2019. https://doi. org/10.1245/s10434-019-07236-8.

3. Fisher B, et al. Tamoxifen for prevention of breast cancer: report of the national surgical adjuvant breast and bowel project P-1 study. J Natl Cancer Inst. 1998;90(18):1371-88.

4. Goss PE, et al. Exemestane for breast-cancer prevention in postmenopausal women. $N$ Engl J Med. 2011;364(25):2381-91.

5. Cuzick J, et al. Anastrozole for prevention of breast cancer in high-risk postmenopausal women (IBIS-II): an international, double-blind, randomised placebo-controlled trial. Lancet. 2014;383(9922):1041-8.

6. Silverman MG, et al. Association between lowering LDL-C and cardiovascular risk reduction among different therapeutic interventions: a systematic review and meta-analysis. JAMA. 2016;316(12):1289-97.

7. Fisher B, et al. Tamoxifen for prevention of breast cancer: report of the national surgical adjuvant breast and bowel project P-1 study. J Natl Cancer Inst. 1998;90(18):1371-88. 
8. Fisher B, et al. Tamoxifen for the prevention of breast cancer: current status of the national surgical adjuvant breast and bowel project P-1 study. J Natl Cancer Inst. 2005;97(22):1652-62.

9. Cigler T, et al. A randomized, placebo-controlled trial (NCIC CTG MAP.2) examining the effects of exemestane on mammographic breast density, bone density, markers of bone metabolism and serum lipid levels in postmenopausal women. Breast Cancer Res Treat. 2011;126(2):453-61.

10. Villani J, Mortensen K. Patient-provider communication and timely receipt of preventive services. Prev Med. 2013;57(5):658-63.

11. Smith SG, et al. Factors affecting uptake and adherence to breast cancer chemoprevention: a systematic review and meta-analysis. Ann Oncol. 2016;27(4):575-90.

12. Holmberg $C$, et al. NRG oncology/national surgical adjuvant breast and bowel project decision-making project-1 results: decision making in breast cancer risk reduction. Cancer Prev Res (Phila). 2017;10(11):625-34.

13. Trivedi MS, et al. Chemoprevention uptake among women with atypical hyperplasia and lobular and ductal carcinoma in situ. Cancer Prev Res (Phila). 2017;10(8):434-41.
14. Brewster AM, et al. A system-level approach to improve the uptake of antiestrogen preventive therapy among women with atypical hyperplasia and lobular cancer in situ. Cancer Prev Res (Phila). 2018;11(5):295-302.

15. Heisey R, et al. Women's views on chemoprevention of breast cancer: qualitative study. Can Fam Phys. 2006;52:624-5.

16. Visvanathan $\mathrm{K}$, et al. Use of pharmacologic interventions for breast cancer risk reduction: American Society of Clinical Oncology clinical practice guideline. $J$ Clin Oncol. 2013;31(23):2942-62.

17. Cuzick J, et al. Preventive therapy for breast cancer: a consensus statement. Lancet Oncol. 2011;12(5):496-503.

Publisher's Note Springer Nature remains neutral with regard to jurisdictional claims in published maps and institutional affiliations. 